\title{
Augmented Reality Based Automotive Engineering and Object Detection System
}

\author{
Jawaria Sallar*, Tehreem Fatima, Sallar Khan, Adil Sheikh, Rafat Ali and Waqar Alam \\ Sir Syed University of Engineering and Technology, Karachi; h.jawaria@yahoo.com, tehreemfatima_97@yahoo.com; \\ sallarkhan_92@yahoo.com, adilshaaikh@gmail.com, rafat.ali500@gmail.com, alam.waqar50@gmail.com
}

\begin{abstract}
Objectives: In this modern era of technology, the learning methods are changing drastically and Augmented Reality (AR) is becoming one of the popular learning methods around the globe for experts and researchers. Methods/Findings: While it has been observed from past studies that the Automotive engineers, researchers, students, and experts need different sources like books, simulation kits, and websites to gather and learn information about the automotive industry and work process of different engine parts, which may is a time taking task and old approach seems to be. In this paper, we have used a 3D creation software blender and solid work for developing live 3D models of different engine parts and tensor flow and machine learning to detect the vehicle objects as well. Application: Our system will provide complete user-friendly control to the user which will assist them to retrieve detailed view of engine parts. These models are a less complex and more attractive way of learning for the user.
\end{abstract}

Keywords: Augmented Reality, Automotive Engineering, Blender, Object Detection, Solid work, 3D Modeling

\section{Introduction}

Augmented Reality (AR) merge virtual world objects with real environments. Over the years, AR has been used in numerous domains for a large number of purposes. In almost all fields, AR is used as a dependable system for performing human Jobs. AR has demonstrated to be useful in growing the accuracy and efficiency of the tasks especially in the domains associated with surgery and air-

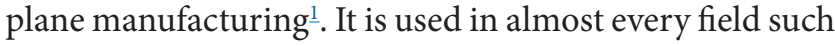
as study, entertainment, games and different industries, etc. There is a little variance between AR and VR. VR is defined as multi-dimensional visual which is totally or partially generated by computer graphics as it changes the whole environment. There are three characteristics of VR that is: 1) Combination of the virtual and real world, 2) Interaction with real time, and 3) Three-dimensional processing. On the other side, the AR technology is a breakdown in optical and video see-through systems.
In optical see-through system, users see the real world through two transmitted monitors. The monitors cover the image of virtual things. The favored AR optical seethrough devices are Microsoft HoloLens, Google Glass and Sony Smart Eyeglass Attach. While on the other side the video see-through seize the real world images through cameras. And they cover the virtual objects in processing and show the developed images in two monitors. Both proceed towards its benefits and drawbacks so the choice depends on your application ${ }^{2}$. There is some contrast between the Web and mobile systems, in terms of the gadgets itself, the network, the end user and the usage context. Mobile gadgets have a comparatively small screen size, and the display also differs in shape from personal computers. There is also a limitation on their input prospect, $\mathrm{CPU}$, storage, bandwidth, and data transmission rate, they have high latency and they are more costly than the World Wide Web (WWW) ${ }^{3}$. Mobile Augmented Reality MAR is a new technology which is used on mobile

*Author for correspondence 
devices as it does more enhancements in mobile devices for users. There are a number of mobile applications based on augmented reality like navigation, sightseeing and tourism, military, promotion and entertainment, etc ${ }^{4}$. Modern science and technology require high quality of engineering education. Developing a more interactive way of getting information is a challenging issue. So the Augmented Reality technologies solve this issue and provide a better platform for interactive educational applications. Augmented Reality is the combination of the real world and $0 \& 1$ 's world data, where 2 and 3 dimension models merge into real-world images in real time and after combining they both make a new world which is more interesting. $\operatorname{In}^{5}$ this modern era, the students are not interested in boring and colorless books they like a visual and attractive way of learning. Moreover, for automotive engineering students, it is very difficult to understand the complicated and intricate parts of a Vehicles Engine without the help of any practical and visual implementation. Students, the person in Automobiles field or any person try to get knowledge about any vehicle engine parts especially the inner and problematic sections of inner parts from different sources but they will not get the exact knowledge about any specific part which they try to seek only due to lots of textual information and less visualization. So they are unable to understand and the knowledge which they get will not help them to interact with real facts. Therefore we are presenting a modern and more visual way of learning for Automotive engineers "AR based Automotive Engineering" (ARAE) is an android based marker less augmented reality application. ARAE uses the markerless augmented reality technology to assist Automobile students to understand and learn internal parts of Engine in a more interactive and visually appealing fashion. This would make users get rid of the old boring books and have it all on their mobile phones. And since we live in an era where the augmented reality has just stepped foot in, we channeled its use in the field other than our information technology. ARAE allows its users to have the best experience of learning while not getting bored during the studies. It's a very innovative way of teaching in the modern world. Our application will provide a better way of learning, save time and effort. AR Based Automotive Engineering visualizes different parts of a car engine i.e., piston, engine block, etc. ARMS are an augmented reality application for medical students. Which is based on marker less augmented reality technology, shows internal organs of the human body in a more interactive way? Students found it more interesting and easy to understand due to its visualization. Car Engine-Augmented Reality shows the part of the engines. You can change the configuration of parts, rotate and remove or hide the parts of models. By turning on transparency you can see the movements and work of internal parts of models. The exposure of innovative technologies helps instructional designers develop learning domain that facilitates learning fast and global use of wireless communication networks and mobile devices has made access to new technologies such as Augmented Reality AR very much easier and has provided remarkable advantages for technology-assisted learning. AR is different from virtual reality which can be defined as a technology allow virtual objects build by computers to be put on physical objects in actual time ${ }^{6}$. There is another teaching software or application for automotive engineers. It teaches how to assemble/disassemble the parts of a vehicle to automotive students. The software teaches the transmission of parts of the engine and tells the set of tools to need to repair any part of a vehicle. It gives a step-by-step instruction of the real process of assembling and dissembling the automobile parts. The student also can go back to the previous instructions if needed. This system consists of two video cameras, a computer with developed software, HMD glasses, and two LCD screens. 3D Modeling provides a more realistic and interesting visualization. 3D Blender provides a platform to convert $2 \mathrm{D}$ into $3 \mathrm{D}$ models. It was a great challenge for computer graphic and vision but now with the help of 3D Blender people can make more realistic images of the interior of buildings, cars, animals, cartoons, etc. Blender has a plethora of various import formats, people use it to translate the initial data into the wave front.obj format, and it is an easy syntax and simple conversion properties. It is a user-friendly platform and easy to import files. As we are working on automobiles the computation of parts and the position of the sensor was a big challenge. VR and AR make it Uncomplicated for us. There are four cases that why we use the augmented reality. The use case helps us to the determination of sensor position, sensor localization and sensor physical application in case of Non-success of visualized result. The basic concept of AR is to operate human visual recognition of the real world by combining real object and computer-generated virtual object in the real world, in 
such a way that they are precisely arranged in real-time ${ }^{7}$. An application for Augmented Reality in the medical field is ultrasound imaging and surgical systems etc. Applications for Augmented Reality in the military to display the real scenes of the battlefield, for military training in citified landscape and training in broad combat scenarios and simulating real-time enemy action, etc., $\stackrel{8}{=}$ here are some restrictions present in this technology. For example, Hsu and Huang said in 2011 that, many people in AR learning practices sympathize that the AR features are good but most people did not accept that some tools are as productive as reading textbooks. They said that using AR systems to get information was not easy. Maybe the reason is that the AR functions are itself is easy to operate but the procedure of sending the image, perceive the images, text and then obtain the meaning of them is time-taking?

\section{Research Methodology}

For the Better accomplishment of the Augmented Reality application, we make some strategy to work smartly. The work is divided into four stages: Designing, Development, Modeling, and Integration. The team started the work parallel for Designing and Development, for this purpose we used Adobe XD and Android studio. Besides this, we created 3D models for the automobile parts on open source platforms i.e., Blender and Solid Work. Finally, we integrate the models in Android Studio to give a complete look of our application.

\subsection{D Model Creation Through}

\subsubsection{Solid Work}

Today in applications and science area, there is also a growing interest and drift in modeling the world in visually three dimensions. To model and Replicate the real world, it is a process of modifying objects' form, motion, Appearance, and other properties according to the significance ${ }^{10}$. To create the $3 \mathrm{D}$ model of the engine parts, first, we start with sketching the piston which is cylindrical as shown in Figures 1, 2. Thereby we will mainly use Revolution tool to model it. We take the front plane of the feature manager on the screen. Then we select the first one to create the sketch by using the smart dimension tool, added horizontal and vertical line to complete the cylinder sketch. Come out of the sketch and select the resolution tool to model the piston. Then we close this sketch and go to features tab, select the extruded cut tool for cutting the piston with the cylinder. This complete the panel likes so and then we save the file as .stl file extension.

\subsubsection{Blender}

We've used the blender to covert the files. To import our file in the Figure 3 we go to the file options and checked the import section and select .STL file. Then we click the .fbx file extension from the export section that is in Figure 4. From the left-hand side on the screen (selected object) we select an export button in the mesh options. The file is then converted into an fbx extension to integrate our android application through the scene from assets.

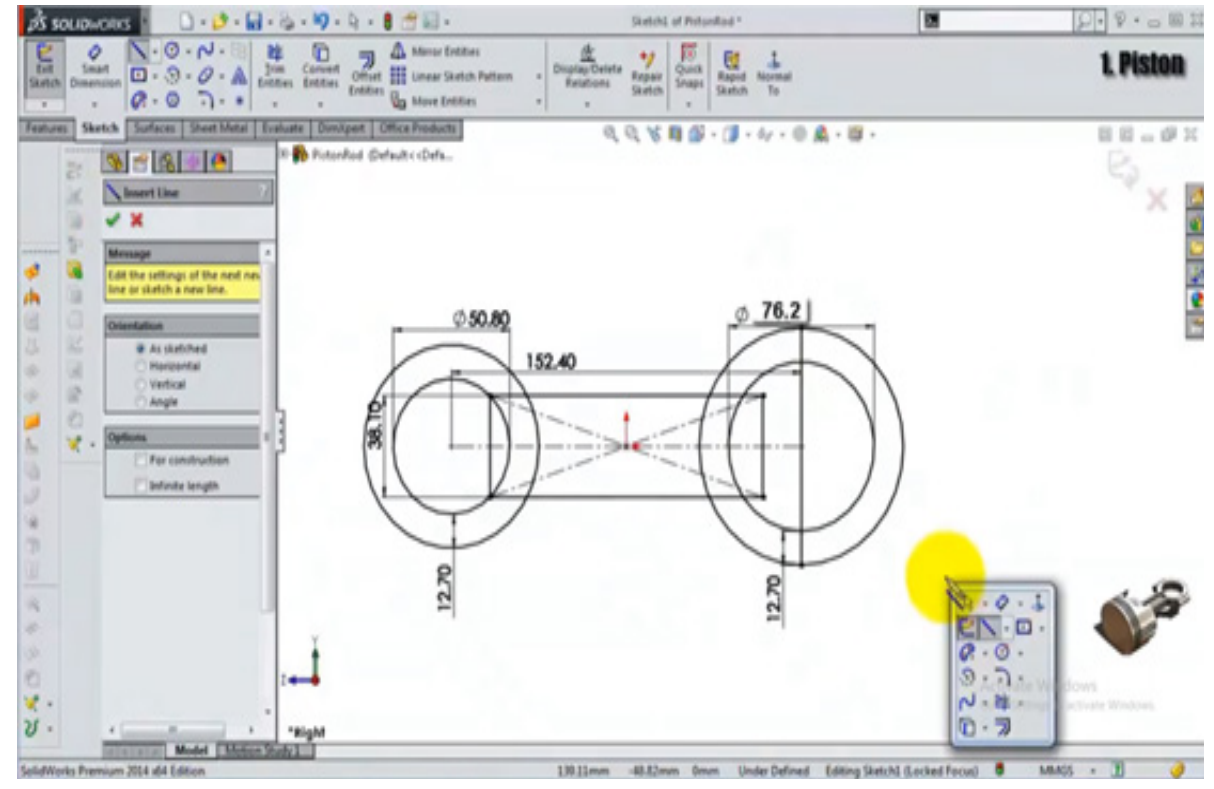

Figure 1. Piston sketching for 3D model. 


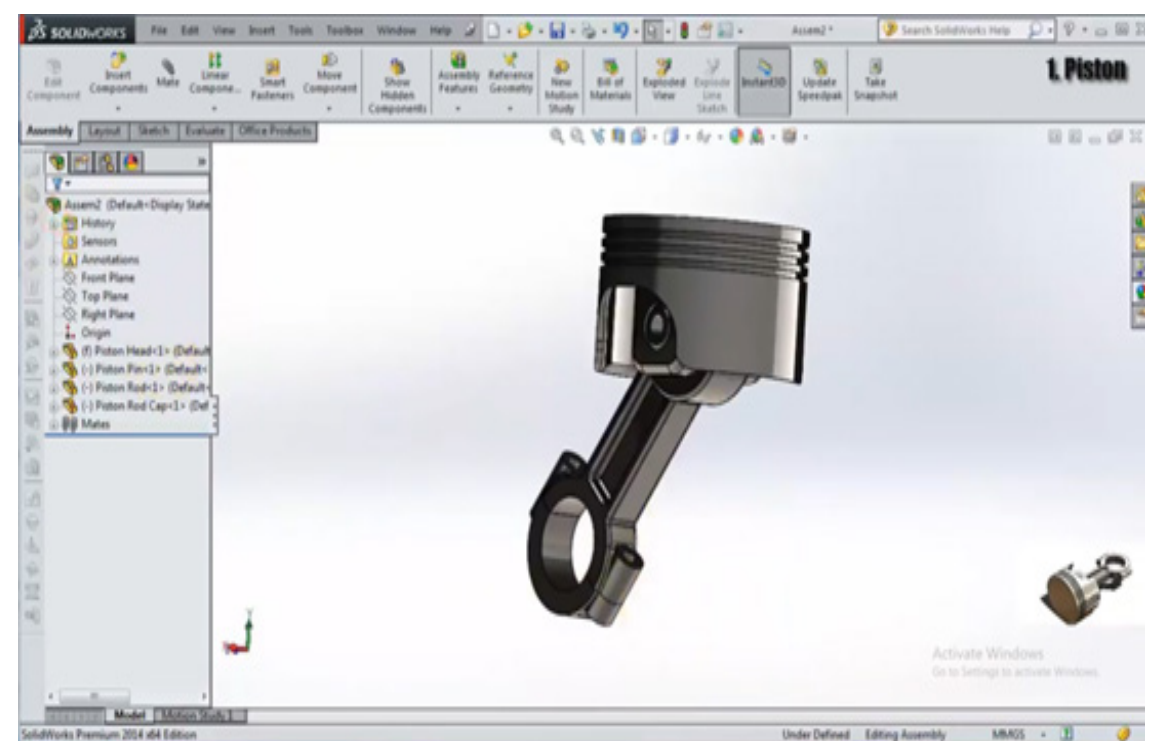

Figure 2. Finalized 3D model for exportation.

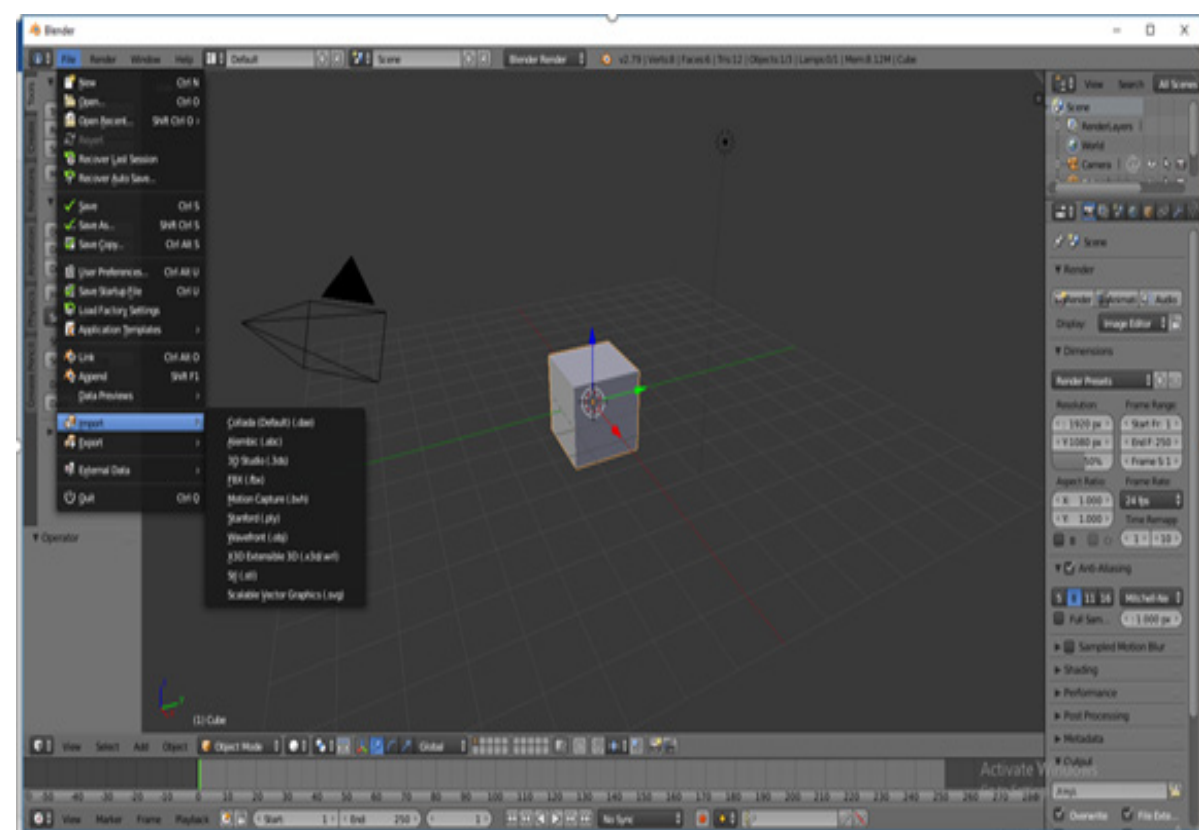

Figure 3. Importing .STL file in blender.

\subsection{Image Detection}

You can clone the repository directly shared https://github. com/azainab/androidTFDetect and adds the files below (but you have to make sure you have all the dependencies mentioned in the following sections added to your system):

1. Go to the downloaded repository folder using a terminal and then type 'gitinit' to activate the repository for git.
2. .pb files in the assets folder (you can download the.pb files from.

3. Note: Select all the files in the android studio and then right click and select git>add. If you face the error 'Failed to finalize session: INSTALL_FAILED_ INVALID_APK: Split lib_slice_0_apk was defined multiple times' just do build >clean project and run again. 


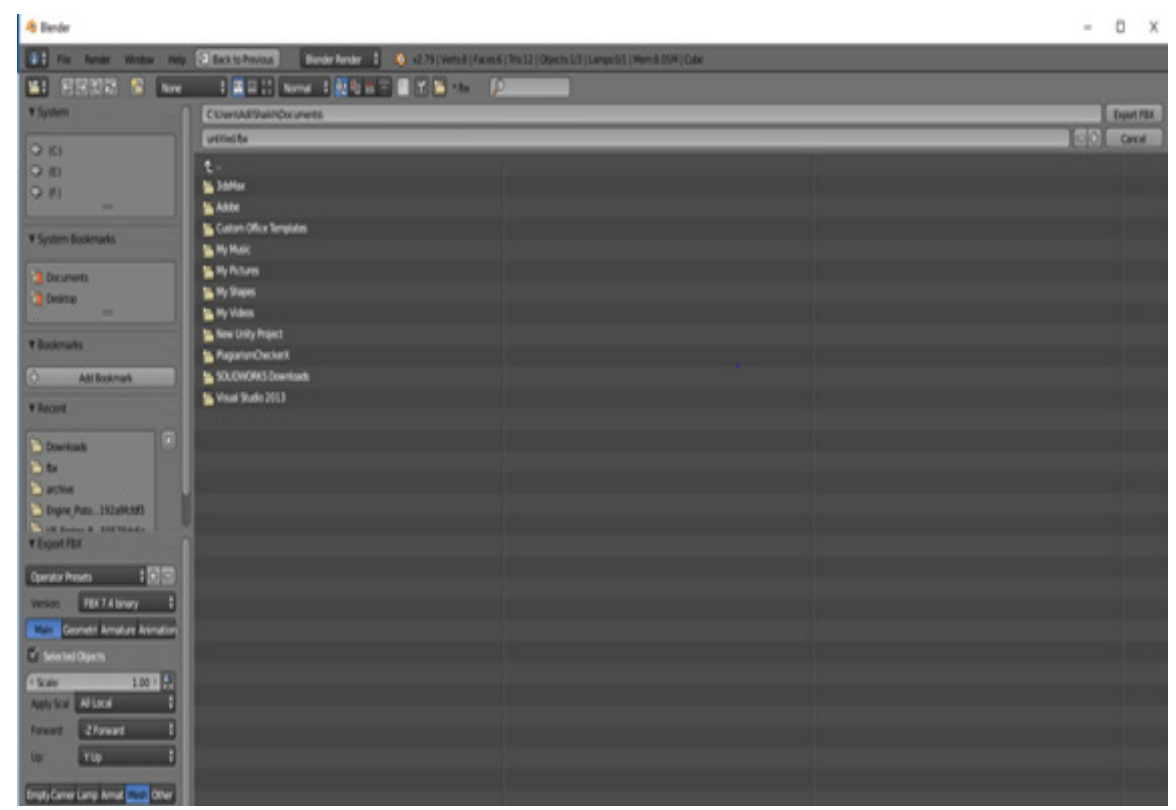

Figure 4. Converting .STL file to .FBX file using export method.

\subsection{Integration}

At this point, we combine all our scattered work and also used some necessary files. The step-by-step procedure is given below:

1. We integrate our user interface UI with firebase by using this line of code of implementation (Implementation 'com.google.firebase: firebase-outh: 16.0.3').

2. We imported our 3D model files with the extension of (.fbx) to the android studio.

3. To give augmented reality experience to our $3 \mathrm{D}$ models we use AR Core platform provided by Google (Implementation 'com.google.ar: core: 1.7.0').

4. To show objects of 3D models we used SCENEFORM TOOL(BITA)(implementation'com.google.ar.sceneform. ux:sceneform-ux:1.7.0'implementation'com.google. ar.sceneform:core:1.7.0').

5. We integrated our image detection app to our augmented reality app to detect the parts of engine.

\section{Results and Discussion}

\subsection{UI Design}

The Login page where students can login to their Id's or can register and create new one and the login information is saved in firebase as shown in Figure 5.

After the login or registration now the user can access to the feature menu as shown in Figure 6.

\subsection{AR Models Labeling}

First you have to find a place to import models and for that you have to move your cell phone and then marker will catch the place to display models. Then through ARCore camera, labeled models will display on screen after just a click on a model and then click on marker as shown in Figure 7. You can rotate and zoom in or zoom out the model according to your requirement. Also you can choose any other model from list as well. First model will remove from screen automatically and next selected model will display on screen.

\subsection{Scanning Images through Models}

Application can scan the model through an image and then display on the screen, as shown in Figure 8. First application will scan the model through an image and match with database to retrieve its information accordingly.

\subsection{Object Detection through Camera}

The Objects are detected through camera using Tensorflow and Machine learning.

Basically the data is retrieve from Google dataset and the Machine learning algorithm extract attributes from input images at real time and uses these attributes to decide the class of the image as shown in Figure 9. 


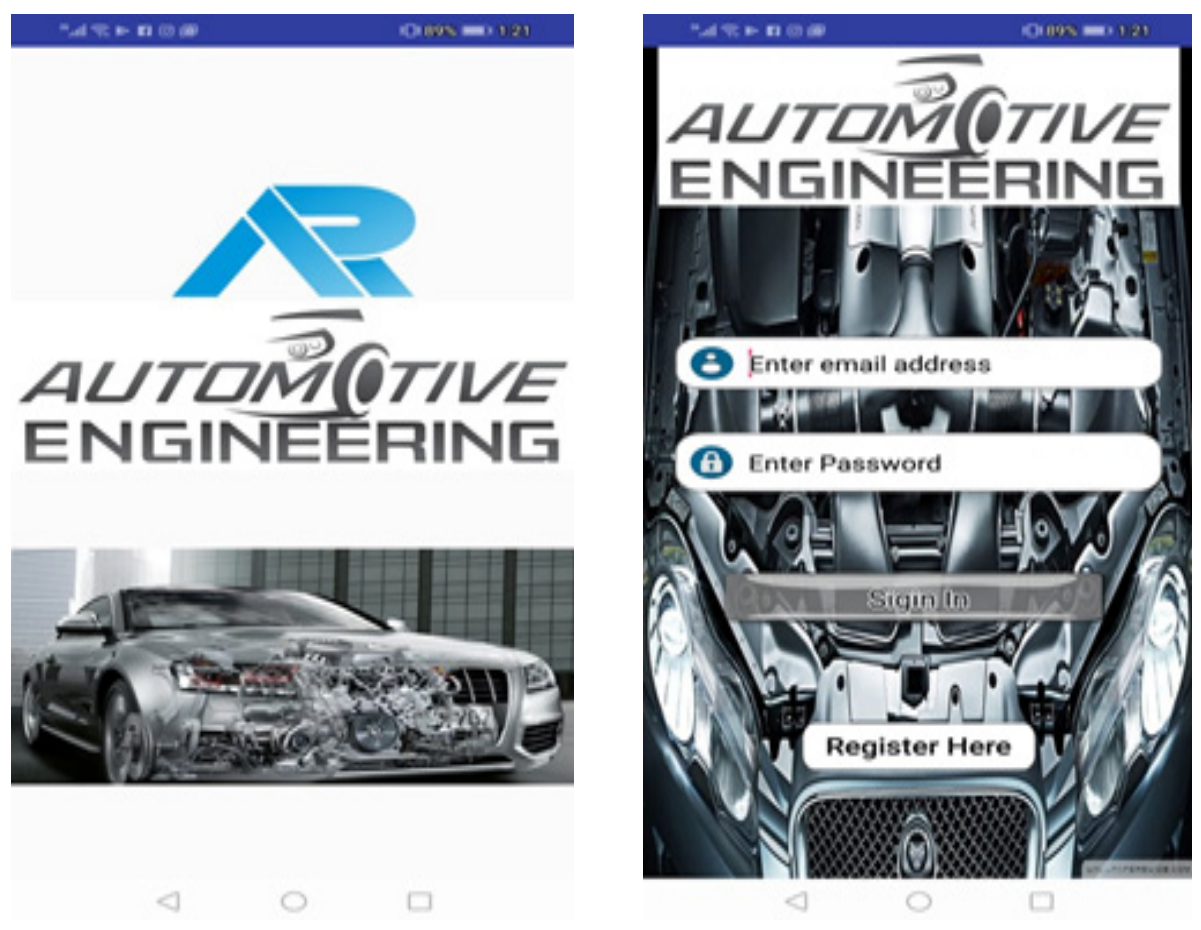

Figure 5. Application Splash page alongside with Sign in Page with Firebase Connectivity.
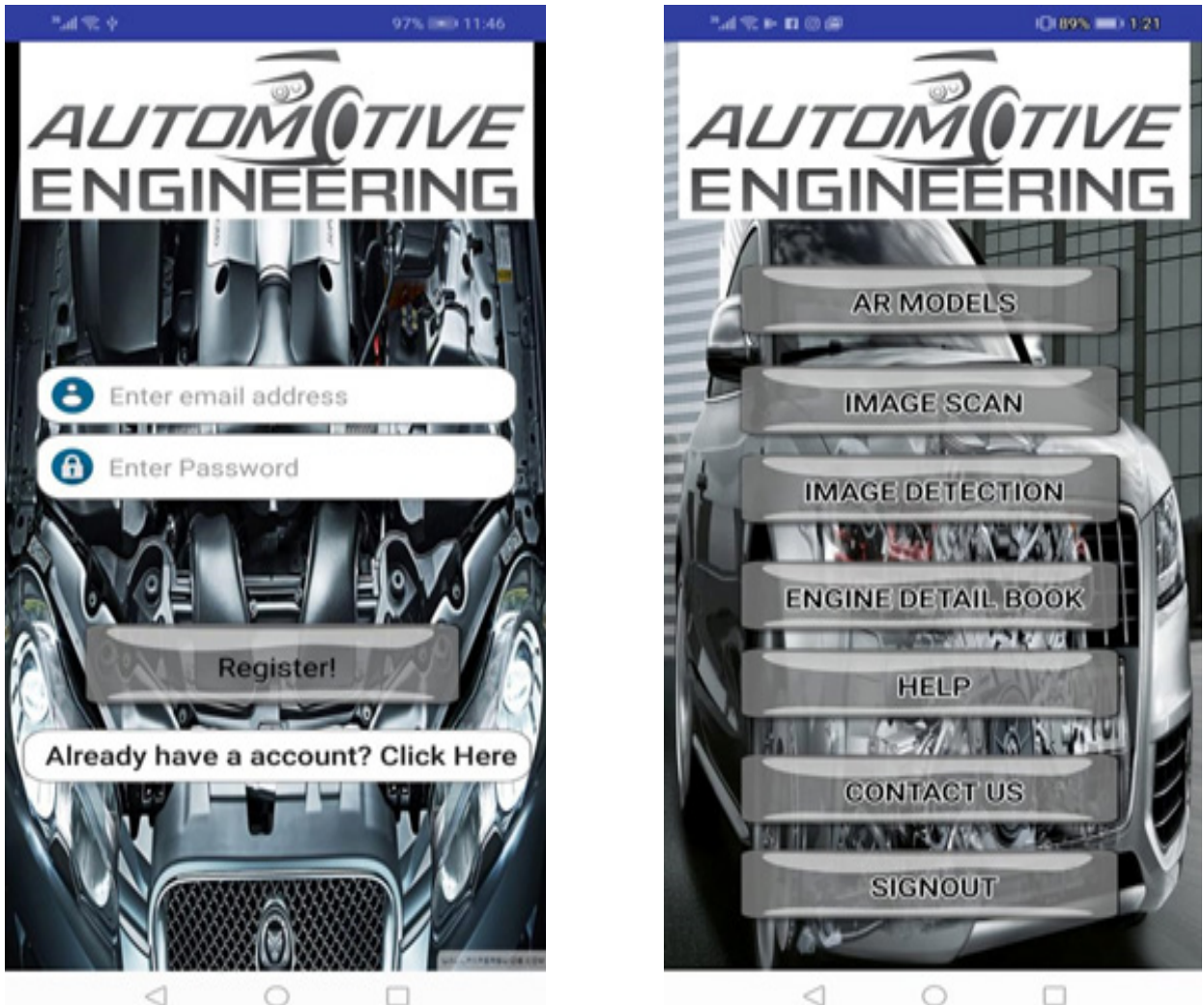

Figure 6. Registration page with firebase connectivity with features menu alongside. 

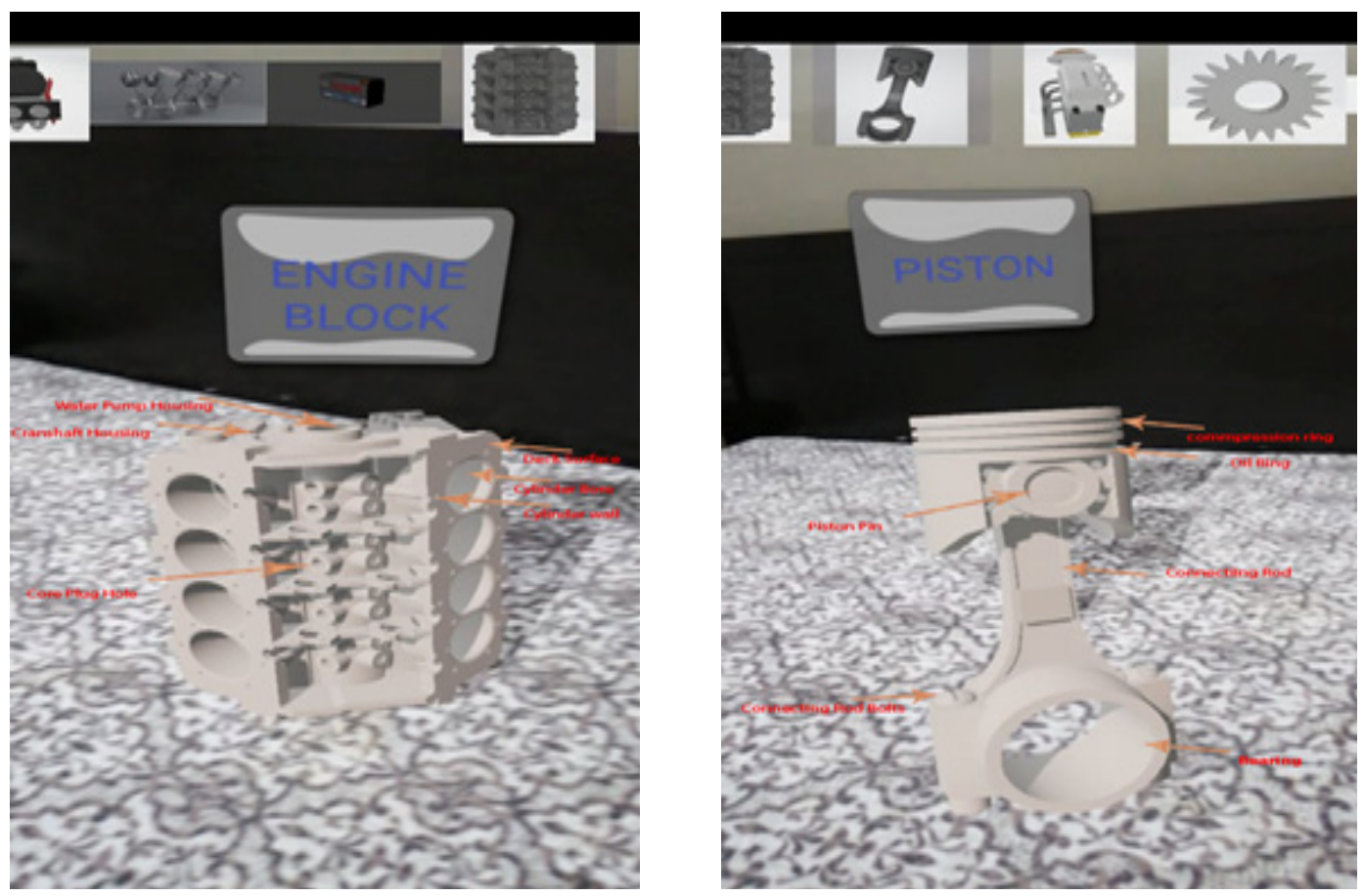

Figure 7. Labeled engine block model with labeled engine piston model on right hand side.
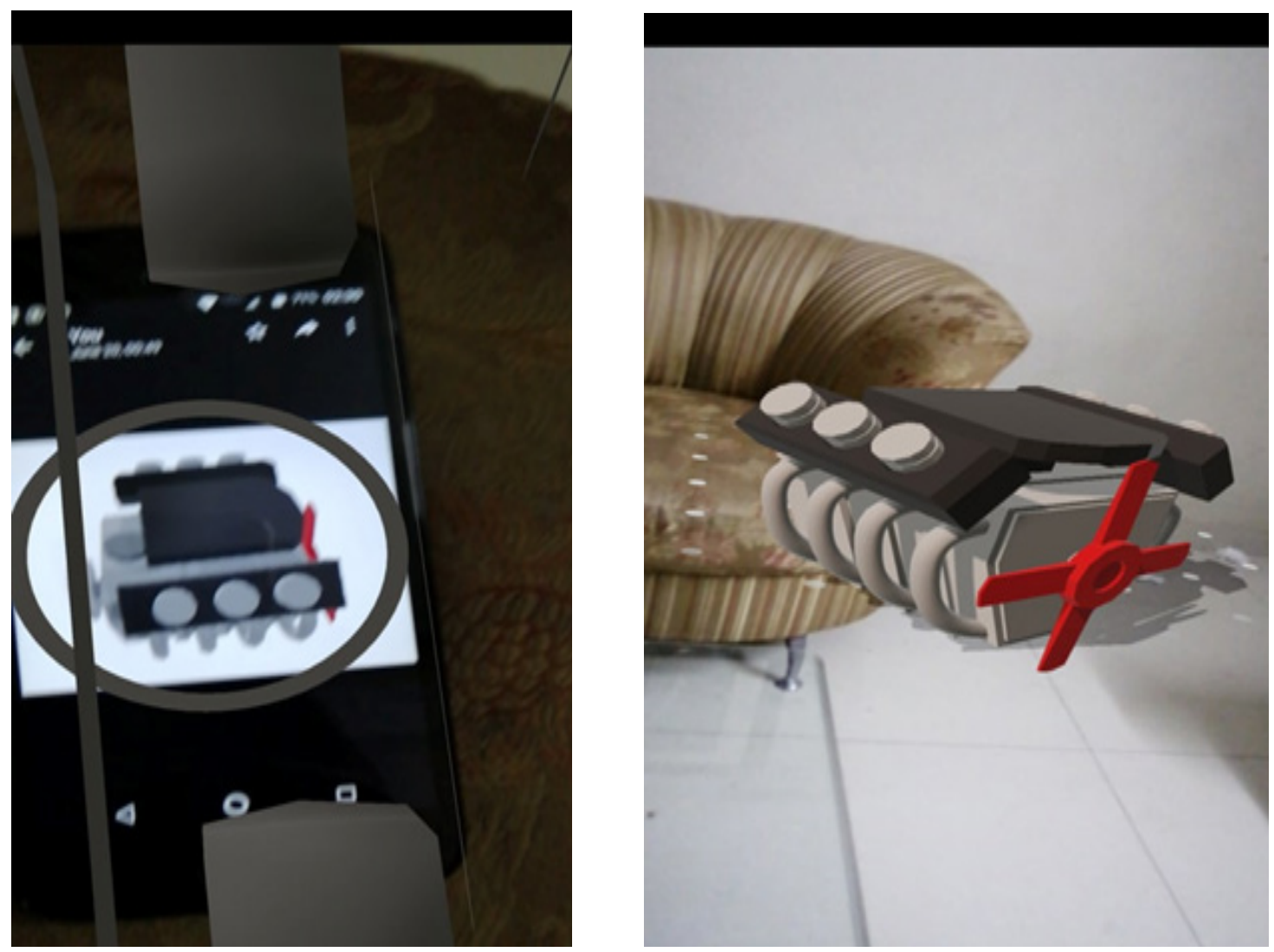

Figure 8. Image scanning with model with AR model displaying the image. 


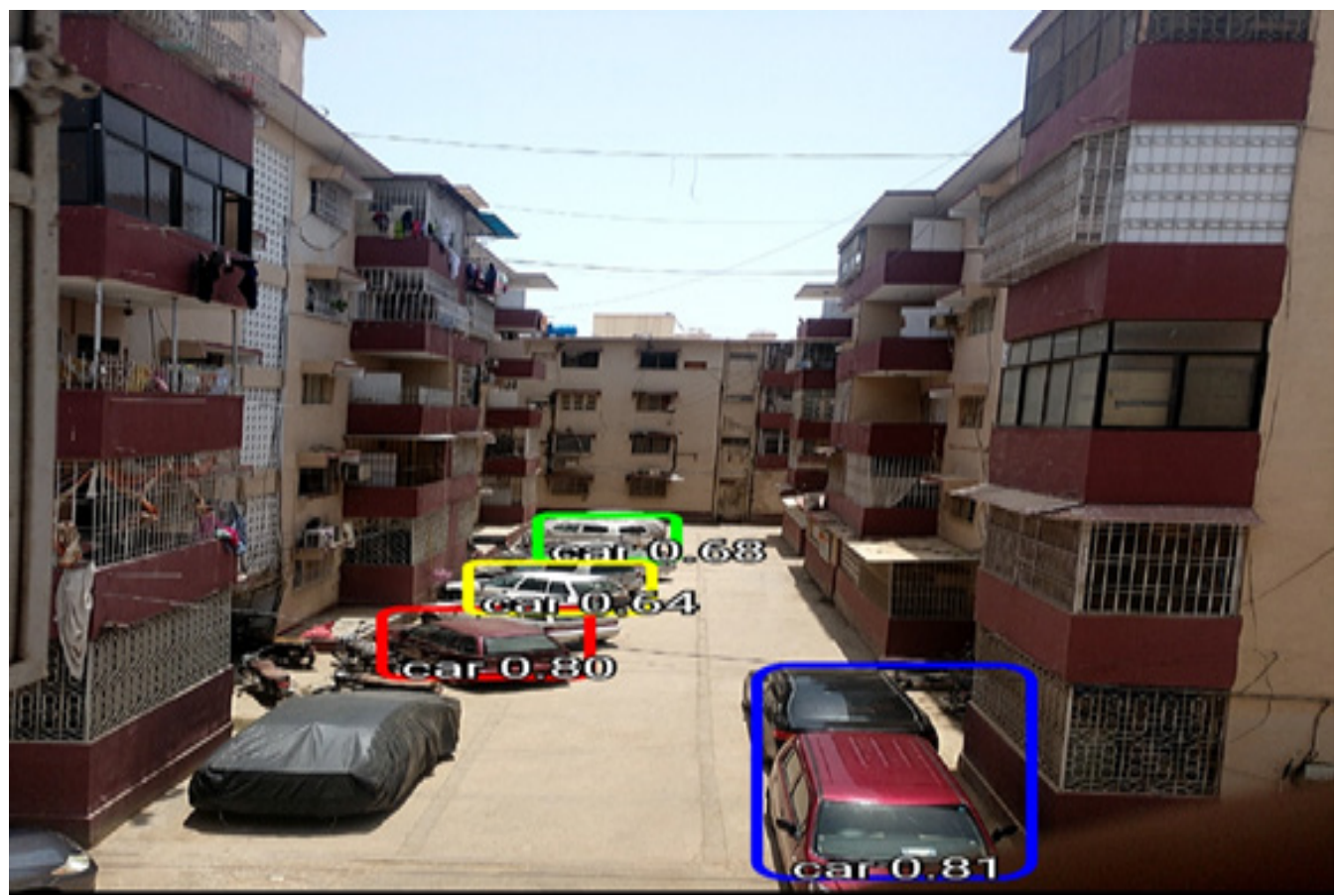

Figure 9. Image detection through tensor flow and machine learning.

\section{Future work}

In future, we will deploy our project in different colleges and universities where Automobile students can easily get facilitate as we will be working on creating the book where the students will scan the picture of different Engine parts and different models and get a 3D Augmented reality model, so that they would better understand the working and mechanism of that part respectively. The project will also facilitate the Auto Showrooms where we can provide a detailed description of their cars so that they can describe the features of car visually and more easily to their Customers. Hence, it will also help out the drivers in the future, as it will provide the guidance to the beginners regarding the car drawbacks and faults inside the Engine resulting in less need of Automobile Mechanics.

\section{Conclusion}

Augmented Reality is an emerging and growing need in the modern era. Hence, it is necessary that we should include this in our daily life and educational institutions for a better understanding and making the studies more interesting and easier for everyone in a way that anyone can easily pick up and understand the concept. This project will help out Automobile engineers in learning and understanding the complicated parts of the engine with the help of 3D and augmented reality for a better experience and visual implementation. The project will provide a platform that can easily explain by detecting the parts and giving the description of those parts of the engine.

\section{References}

1. Deshmukh SS, Joshi CM, Patel RS, Gurav YB. 3D Object tracking and manipulation in augmented reality. International Research Journal of Engineering and Technology. 2018; 5(1):287-9.

2. Faath A, Steinmetz C, Anderl R, Faath A. Application of virtual and augmented reality in automotive measurement processes presenting author. International Science Fiction Prototyping Conference. Bruges, Belgium; 2017. p. 1-23.

3. Oinas-kukkonen H. Developing successful mobile applications; 2003.

4. Sudarshan SK. Presented to the faculty of the department of software engineering San José State University in partial fulfillment of the requirements for the Degree Master of Science. Available from: https://cmpe.sjsu.edu/

5. Farkhatdinov I, Ryu J. Development of educational system for automotive engineering based on augmented reality. International Conference Engineering; 2017. 
6. Ozdemir M, Sahin C, Arcagok S, Demir MK. The effect of augmented reality applications in the learning process: A meta analysis study. Eurasian Journal of Education Research. 2018; 18:1-22. https://doi.org/10.14689/ejer.2018.78.6

7. Rosanny S, Volker C. Object-based mobile augmented reality for a 3D Model; 2018. p. 646-55.

8. Mekni M, Lemieux A. Augmented reality: Applications, challenges and future trends. Applied Computer Science. 2014. p. 205-14.
9. Saidin NF, Halim NDA, Yahaya N. A review of research on augmented reality in education: Advantages and applications. International Education Studies. 2015; 13(8):1-8. https://doi.org/10.5539/ies.v8n13p1

10. Luan X, Xie Y, Ying L, Wu L. Research and development of 3D modeling. IJCSNS International Journal of Computer Science and Network Security. 2008; 8(1): 49-53. 